\title{
Truncated Vector Lattices
}

\author{
Richard N. Ball ${ }^{1}$ \\ Department of Mathematics, University of Denver, Denver CO 80210, USA \\ rball@du.edu
}

In analysis, truncation is the operation of replacing a nonnegative real-valued function $a(x)$ by its pointwise meet $a(x) \wedge 1$ with the constant 1 function. A vector lattice $A$ is said to be closed under truncation if $a \wedge 1 \in A$ for all $a \in A^{+}$. Note that $A$ need not contain

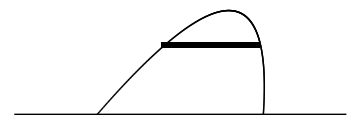
1 itself.

Truncation is fundamental to analysis. To give only one example, Lebesgue integration generalizes beautifully to any vector lattice of real-valued functions on a set $X$, provided the vector lattice is closed under truncation. But vector lattices lacking this property may have integrals which cannot be represented by any measure on $X([5])$. Nevertheless, when the integral is formulated in a context broader than $\mathbb{R}^{X}$, for example in pointfree analysis, the question of truncation inevitably arises.

What is truncation, or more properly, what are its essential properties? In this talk we answer this question by providing the appropriate axiomatization, and then go on to present several representation theorems. The first is a direct generalization of the classical Yosida representation of an archimedean vector lattice with order unit. The second is a direct generalization of Madden's pointfree representation of archimedean vector lattices. If time permits, we briefly discuss a third sheaf representation which has no direct antecedent in the literature.

However, in all three representations the lack of a unit forces a crucial distinction from the corresponding unital representation theorem. The universal object in each case is some sort of family of continuous real-valued functions. The difference is that these functions must vanish at a specified point of the underlying space or locale or sheaf space. With that adjustment, the generalization from units to truncations goes remarkably smoothly. (Details are available from the author [1], 2].)

\section{Truncation Defined}

Definition 1. A truncation on a vector lattice $A$ is a unary operation $A^{+} \rightarrow A^{+}$, written $a \longmapsto \bar{a}$, which has the following three properties for all $a, b \in A^{+}$.

(T1) $a \wedge \bar{b} \leq \bar{a} \leq a$

(T2) If $\bar{a}=0$ then $a=0$.

(T3) If $n a=\overline{n a}$ for all $n$ then $a=0$.

A truncated vector lattice, or trunc for short, is a vector lattice equipped with a truncation. A trunc $A$ is said to be unital if it contains an element $u \geq 0$ such that $a=a \wedge u$ for all $a \in A^{+}$. $A$ truncation morphism is a vector lattice morphism which preserves the truncation. We use $\mathbf{T}$ to designate the category of truncs and trunc morphisms.

The first axiom expresses the desideratum $a \wedge(b \wedge 1) \leq a \wedge 1 \leq a$, the second expresses the idea that no positive element should be disjoint from the unit, and the third can be read as banning elements which are infinitesimal with respect to the unit. 


\section{The Pointed, or Yosida Representation}

The subdirectly irreducible truncs, which coincide with the simiple truncs, i.e., those truncs $A$ having only trivial quotients $A$ and 0 , are the totally ordered truncs. Such truncs have a unit $u$, which occupies a least nontrivial convex subgroup. Birkhoff's Theorem looks like this.

Proposition 1. Any trunc is isomorphic to a subdirect product of totally ordered truncs, exactly one of which is the 0 trunc.

But we can do much better, at least in the subcategory AT of archimedean truncs.

Theorem 1. For each archimedean trunc $A$ there is a pointed compact Hausdorff space $(X, *)$, a trunc $\widehat{A}$ in $\mathcal{D}_{*} X$ which separates points from closed sets in $X$, and an isomorphism $A \rightarrow$ $\widehat{A}$. The space is unique up to pointed homeomorphism with respect to its properties, and the representation is fully functorial.

What is $\mathcal{D}_{*} X$ you ask? It is the family of continuous extended-real valued functions $X \rightarrow$ $\overline{\mathbb{R}}=\mathbb{R} \cup\{ \pm \infty\}$ which are almost finite, i.e., take on the values $\pm \infty$ on a nowhere dense set, and which vanish at the designated point $* \in X$. This family forms a lattice under the pointwise order, but it is not closed under the obvious addition. A subset which is closed under the trunc operations is called a trunc in $\mathcal{D}_{*} X$. (In spite of the fact that every beginning calculus student is sure he can add or subtract functions with only a few infinities, the truth is that it is a mess. But see the next representation.)

If a trunc is not unital, is there at least some best way to attach a unit? This is an important question because the unital archimedean truncs constitute the famous and well-studied category $\mathbf{W}$, which appears in this context as a full subcategory of AT. In fact, these are exactly the archimedean truncs for which the designated point $*$ is isolated in its spectrum $X$. The question, then, is whether $\mathbf{W}$ is reflective in $\mathbf{A T}$.

Proposition 2. W is a bireflective subcategory of $\mathbf{A T}$. The reflection of a given trunc $A \subseteq$ $\mathcal{D}_{*} X$ is obtained by adding a new isolated point to $X$, say $Y=X \cup\left\{*_{Y}\right\}$, extending the functions of $A$ from $X$ to $Y$ by setting them to 0 at $*_{Y}$, and then adjoining the function which is constantly 1 on $X$ and 0 at $*_{Y}$.

\subsection{Does Every Archimedean Vector Lattice Admit a Truncation?}

A celebrated example of Conrad and Martinez ([3]) presents an archimedean vector lattice which cannot be embedded in any vector lattiice having a unit. If this vector lattice admitted a truncation, its $\mathbf{W}$-reflection would constitute just such an embedding. So the answer to the question posed in the subsection title is no. However, examples of this type seem quite exotic.

\section{The pointfree, or Madden representation}

Just as in the unital case of $\mathbf{W}$, passage to the pointfree context cleans up the representation, eliminating all reference to functions taking on the values $\pm \infty$. But here again, the functions must all vanish at a specified point.

A pointed frame $(L, f)$ consists of a completely regular frame $L$ together with a designated point $f: L \rightarrow 2$. For example, the pointed frame of the reals, written $\mathcal{O}_{0} \mathbb{R}$, has the frame $\mathcal{O} \mathbb{R}$ of open subsets of $\mathbb{R}$ together with the frame map $\mathcal{O} \mathbb{R} \rightarrow 2$ corresponding to the constant 0 function on $\mathbb{R}$. Pointed frame maps are simply frame morphisms which commute with the 
points. The family $\mathcal{R}_{0} L$ of all pointed frame maps $\mathcal{O}_{0} \mathbb{R} \rightarrow L$ is precisely the frame counterpart of the family of all locale maps $L \rightarrow \mathbb{R}$ which vanish at 0 , and as such inherits a natural trunc structure from $\mathcal{R} L$, the familiar $\mathbf{W}$-object of all frame maps $\mathcal{O} \mathbb{R} \rightarrow L$.

Theorem 2. For any divisible archimedean trunc A there exists a regular Lindelof frame $L, a$ subtrunc $\widehat{A} \subseteq \mathcal{R}_{0} L$ which is cozero dense, and a trunc isomorphism $A \rightarrow \widehat{A}$. The frame is unique up to isomorphism with respect to its properties, and the representation is fully functorial.

Just as in the Madden representation of $\mathbf{W}$-objects, the frame $L$ used in the representation of a trunc $A$ is the frame of kernels of $A$, in this case the $\mathbf{T}$-kernels. But the construction cannot by its nature apply outside archimedean truncs, and an additional axiom must be added to the definition of truncation so as to restrict the objects to archimedean $\ell$-groups.

\section{The Sheaf Representation}

The sheaf representation handles the most general sort of trunc - axiom (T3) is relaxed so as to permit infinitesimals. It also gives the tightest representation, in the sense that the given trunc is often isomorphic to the trunc of global sections of its sheaf. The trade-off is that the data required by the representation is more extensive. And the trivial truncation $\bar{a}=a$ is now in play.

Definition 2. Let $(X, *)$ be a pointed space. A presheaf of truncs over $X$ is a contravariant functor $S: \mathcal{O} X \rightarrow \mathbf{T}$ satisfying these three conditions.

1. $S(U)$ is unital if $* \notin \operatorname{cl} U$.

2. For all $U \in \mathcal{O} X$ and for all $a_{i} \in S(U)^{+}$such that $a_{i} \ominus 1 \neq 0$ and $a_{1} \wedge a_{2}=0$, there exist $V_{i} \in \mathcal{O} X$ such that $a_{i} \ominus 1 \mid V_{i}=0$ and $V_{1} \cup V_{2}=U$.

3. For all $U \in O \not X$ such that $* \in U$, and for all $a \in S(U)^{+}$such that $a \ominus 1 \neq 0$, there exist $V \in \mathcal{O} X$ such that $* \in V \subseteq U$ and $a \ominus 1 \mid V=0$.

$S$ is a sheaf of truncs if, in addition, it has the following properties for any family $\left\{U_{i}: i \in I\right\} \subseteq$ $\mathcal{O} X$ with $U \equiv \bigcup_{I} U_{i}$.

(4) If $s, t \in S(U)$ satisfy $s\left|U_{i}=t\right| U_{i}$ for all $i$ then $s=t$.

(5) For every family $\left\{s_{i} \in S\left(U_{i}\right): i \in I\right\}$ of sections such that $s_{i}\left|\left(U_{i} \cap U_{j}\right)=s_{j}\right|\left(U_{i} \cap U_{j}\right)$ for all $i, j \in I$, there exists a single section $s \in S(U)$ such that $s \mid U_{i}=s_{i}$ for all $i$.

Property (1) asserts that the non-unital behavior of a trunc is confined to the designated point $*$ of its spectrum. Property (2) asserts that disjoint members of a given section can be separated by disjoint open sets. Property (3) asserts that a positive element of a section containing $*$ must be less than any given $\varepsilon>0$ on a neighborhood of $*$.

With every trunc there is a naturally associated presheaf over its spectrum. It is rarely a sheaf.

Proposition 3. Let $A$ be a trunc with spectrum $(X, *)$. Then the assignment $S(U) \equiv A / P(U)$, where $P(U)$ is the polar

$$
P(U)=\bigcap_{U(a) \subseteq U} a^{\perp} \cap \bigcap_{V(a) \subseteq U} \bigcap_{n} a \ominus \frac{n}{n+1}^{\perp \perp}, a \in A^{+},
$$

constitutes a presheaf of truncs over $X$ whose trunc of global sections is $A$. 


\subsection{Spaces of Local Truncs}

The simplest truncs are those with one or two elements in their spectra. These are the building blocks of all truncs.

Definition 3. A trunc $A$ is called local if its spectrum $X$ is either $\{*\}$, in which case $A$ is trivial with truncation $\bar{a}=a$, or $X$ is $\{*, x\}$.

Nothing can be said about the structure of trivial truncs, for any vector lattice is a trunc when equipped with its trivial truncation $\bar{a}=a$. But a great deal can be said about the structure of truncs with a single non-* value.

Recall that a vector lattice $A$ is said to be a lex extension of one of its convex subl-vector lattices $B$ if $B$ contains every minimal prime of $A$ (4]). Every such $A$ contains a smallest such $B$, referred to as the lex kernel of $A$ and written lex $A$. Recall also that any $a \in A^{+} \backslash \operatorname{lex} A$ is special, i.e., has a single value.

Proposition 4. The following are equivalent for a nontrivial trunc A.

1. A is local.

2. A is unital and its unit lies outside its lex kernel.

3. $A$ is unital and its unit is special.

4. If $a \wedge b=0$ then $a \ominus 1=0$ or $b \ominus 1=0$.

5. lex $A$ is a proper truncation ideal.

Fix a pointed compact Hausdorff space $(X, *)$.

Definition 4. A space of local truncs over $X$ is a pair $(E, \pi)$, where $E$ is a space and $\pi$ is a continuous surjection $E \rightarrow X$ with these properties.

1. $E_{x} \equiv \pi^{-1}(x)$ is a local trunc for each $x \in X$, and $E_{x}$ is trivial iff $x=*$.

2. $\pi$ is a local homeomorphism.

3. All operations (subtraction, meet, and truncation) are continuous.

A space of local truncs $(E, \pi)$ arises naturally from a presheaf $S: \mathcal{O} X \rightarrow \mathbf{T}$ in standard fashion. Furthermore, each space $(E, \pi)$ of local truncs over $(X, *)$ generates a sheaf $R: \mathcal{O} X \rightarrow$ T of truncs, simply by letting $R(U)$ be the trunc of sections $U \rightarrow E$, and by letting $R(U \hookrightarrow V)$ be the restriction map $R(V) \rightarrow R(U)$. We refer to $R$ as the sheafification of $S$. When $S$ is the presheaf associated with a given trunc $A$, we refer to $R$ as the sheaf associated with $A$.

Let $A$ be a trunc with spectrum $(X, *)$, and let $R: \mathcal{O} X \rightarrow \mathbf{T}$ be the sheaf associated with $A$. The trunc $B$ of global sections of $R$ contains a copy of $A$, of course, since $A$ is $S(X)$ and the canonical $i_{X}: S(X) \rightarrow R(X)$ is injective. When $A$ is identified with its image, each element of $B$ is approximated by the elements of $A$, in the sense that, for every $x \in X$ and $b \in B$ there is some neighborhood $U$ of $x$ and element $a \in A$ such that $b|U=a| U$. We describe this state of affairs by saying that each member of $B$ is locally equal to a member of a.

Theorem 3. Any trunc $A$ is isomorphic to a subtrunc of the trunc $B$ of global sections of the sheaf $R$ associated with $A$. When identified with its image under such an isomorphism, $A$ has the same spectrum as $B$ does, and every member of $B$ is locally equal to a member of $A$. Furthermore, $B$ is unique up to isomorphism over $A$ with respect to its properties. 


\section{References}

[1] R. N. Ball. Truncated abelian lattice-ordered groups I: the pointed (Yosida) representation. 2013. submitted.

[2] R. N. Ball. Truncated abelian lattice-ordered groups II: the pointfree (Madden) representation. 2013. submitted.

[3] P. Conrad and J. Martinez. Settling a number of questions about hyper-archimedean lattice-ordered groups. Proc. Am. Math. Soc., 109:291-296.

[4] M. R. Darnel. Theory of lattice-ordered groups, volume 187 of Pure and Applied Mathematics. Marcel Dekker, Inc., 1995.

[5] B. R. F. Jefferies and D. H. Fremlin. An indecomposable Daniell integral. Proc. Am. Math. Soc., 101:647-651, 1987. 